\title{
Ligadura videotoracoscópica da persistência do canal arterial
}

\author{
Gladyston Luiz de Lima SOUTO*, Renan Catharina TINOCO*, Augusto Claúdio de A. TINOCO*, \\ Celme da Silva CAETANO*, José Bruno SOUZA* , Ary Getúlio de PAULA*, Marco Antonio TEIXEIRA*, \\ Márcio Roberto CARVALHO*, Antonio Carlos BOTELHO*, Sandro B. P. COELHO*, Hanry B. SOUTO*
}

RBCCV 44205-497

Souto G L L, Tinoco R C, Tinoco A C A, Caetano C S, Souza J B, Paula A G, Teixeira M A, Carvalho M R, Botelho A C, Coelho S B P, Souto H B - Ligadura videotoracoscópica da persistência do canal arterial. Rev Bras Cir Cardiovasc 2000; 15(2): 154-9.

RESUMO: Objetivo: Demonstrar a técnica de fechamento da Persistência do Canal Arterial (PCA), destacando a simplicidade e singularidade do método.

Casuística e Método: No período de março de 1994 a novembro de 1999, 40 pacientes (pac), com idade entre 8 meses e 17 anos e predominando o sexo masculino, foram submetidos a operação de fechamento da PCA através de videotoracoscopia. O paciente é colocado em decúbito lateral direito, sob anestesia geral com intubação seletiva do pulmão direito. São usados quatro trocateres: um de $3 \mathrm{~mm}$ no $3^{\circ}$ espaço intercostal (EIC) esquerdo na linha axilar anterior e outro trocater de $5 \mathrm{~mm}$ no $3^{\circ}$ (EICE) na linha axilar média. A ótica 30 graus infantil é introduzida em um trocater de $3 \mathrm{~mm}$ no $5^{\circ}$ EICE na linha axilar posterior e outro trocater de $5 \mathrm{~mm}$ no $5^{\circ}$ EICE na linha axila anterior. A identificação do canal é feita tendo como parâmetro anatômico os nervos frênico e vago. Após o isolamento, o canal é duplamente clipado ou ligado com fio de algodão grosso com nó interno. Terminado o procedimento, o pulmão é expandido sob visão direta, não havendo necessidade de drenar o tórax.

Resultados: Obtivemos sucesso inicial em 37 pac (92,5\%), sendo necessária reversão para operação convencional em 3 pac $(7,5 \%)$. Não houve óbito nem complicações e o tempo médio de hospitalização foi de 48 horas.

Conclusão: Acreditamos ser o procedimento eficaz, seguro, com pequeno índice de complicações, custo baixo e uma curva de aprendizado também pequena.

DESCRITORES: Procedimentos cirúrgicos cardíacos, métodos. Canal arterial, cirurgia. Toracoscopia, métodos. Cirurgia minimamente invasiva, métodos.

\section{INTRODUÇÃO}

A Persistência do Canal Arterial (PCA) corresponde a 5 a $10 \%$ das cardiopatias congênitas (1). Após 1 ano de vida somente $0,6 \%$ fecham espontaneamente (2).

O fechamento cirúrgico da PCA foi sugerido pela primeira vez à Academia de Cirurgia da Filadélfia, em 1907, por John MURO (3); GRAYBIEL et al. (4), em 1938, fizeram a primeira tentativa de ligadura em uma jovem de 22 anos com endocardite, porém foram GROSS \& HUBBARD ${ }^{(5)}$, em 1939, os primeiros a executar a ligadura bem sucedida em uma criança de 7 anos de idade com insuficiência cardíaca crônica, inaugurando a era moderna de cirurgia para correção dos defeitos cardíacos congênitos.

Trabalho realizado no Serviço de Cardiologia do Hospital São José do Avaí. Itaperuna, RJ, Brasil. Apresentado ao 26ํㅡㄹ Congresso Nacional de Cirurgia Cardíaca. Fortaleza, CE, 8 a 10 de abril de 1999.

*Do Serviço de Cardiologia do Hospital São José do Avaí.

Endereço para correspondência: Gladyston Luiz Lima Souto. Rua Lenira Tinoco Calheiros, 242. Centro. Itaperuna, RJ, Brasil. CEP 28300-000. Tel. (24) 824-4191. Fax (24) 824-3694. e-mail: gladyston@tic.psi.br 
Souto G L L, Tinoco R C, Tinoco A C A, Caetano C S, Souza J B, Paula A G, Teixeira M A, Carvalho M R, Botelho A C, Coelho S B

P, Souto H B - Ligadura videotoracoscópica da persistência do canal arterial. Rev Bras Cir Cardiovasc 2000; 15(2): 154-9.

Em 1967, PORSTMANN et al. (6, 7) trataram a PCA por cateterismo intervencionista, porém, no final dos anos 70, RASHKIND et al. (8) introduziram um novo sistema oclusivo que foi bastante difundido. Houve, depois, uma popularização do uso de umbrellas e de molas ou coil de Gianturco $(9,10)$. Embora estes métodos sejam menos invasivos, ainda são muito limitados $(8,11-13)$.

Em 1993, uma nova técnica de ligadura da PCA por videotoracoscopia foi descrita por LABORDE et al. $(14,15)$, inaugurando, assim como GROSS \& HUBBARD em 1939, uma nova era da correção cirúrgica dos defeitos congênitos.

O objetivo deste trabalho é descrever nossa experiência com o fechamento da PCA por toracoscopia.

\section{CASUÍSTICA E MÉTODOS}

No período de março de 1994 a novembro de 1999, 40 pacientes com idade entre 8 meses e 17 anos, predominando o sexo masculino, foram submetidos a cirurgia de fechamento da PCA, através de videotoracoscopia. Em nenhum paciente havia outra doença congênita concomitante.

\section{Técnica Operatória}

O paciente é colocado em decúbito lateral direito, sob anestesia geral, com intubação seletiva direita. É usado um trocater de $3 \mathrm{~mm}$ no 3 o espaço intercostal esquerdo (EICE), na linha axilar anterior e um trocater de $5 \mathrm{~mm}$ no $3^{\circ}$ EICE na linha axilar média, ambos para trabalho do cirurgião. Uma ótica de 30 graus infantil é introduzida em um trocater de $3 \mathrm{~mm}$ no 50 EICE na linha axilar posterior e outro trocater de $5 \mathrm{~mm}$ no $5^{\circ}$ EICE na linha axilar anterior, para 0 auxiliar (Figura 1).

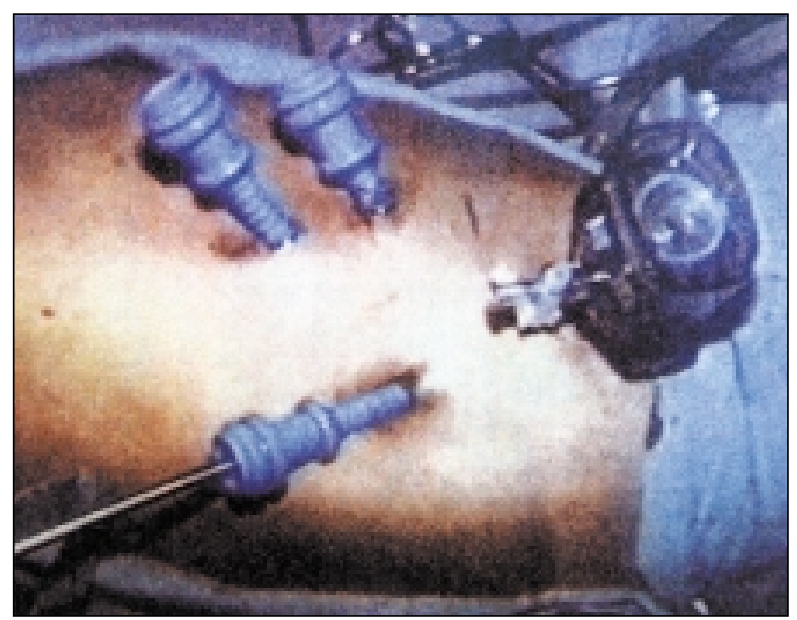

Fig. 1 - Mostrando as posições dos trocateres.
A pleura mediastinal é aberta longitudinalmente entre o nervo frênico e o nervo vago. O nervo vago é dissecado até o ponto de saída do nervo laríngeo recurrente que contorna a parede posterior do canal arterial. A dissecção continua no istmo aórtico, descendo junto à aorta, às vezes ligando algumas veias que cruzam esta região. $O$ saco pericárdico pode estender-se sobre a face anterior do canal, obrigando o auxiliar a afastá-lo para a linha média. A dissecção da parede posterior do canal é a parte mais crítica da operação devido ao risco de sangramento (Figuras 2 e 3 ). Todo o tecido frouxo que envolve o canal é liberado com dissecção com tesoura (Figuras 2, 3, 4 e 5).

O canal é contornado com pinça de Maryland e ligado com 2 clips de titânio de $1 \mathrm{~cm}$ (Figuras 6 e 7).

Em alguns casos, temos feito ligadura com fio de algodão grosso contornando o canal, dando quatro nós internos. Utilizamos esta técnica quando o

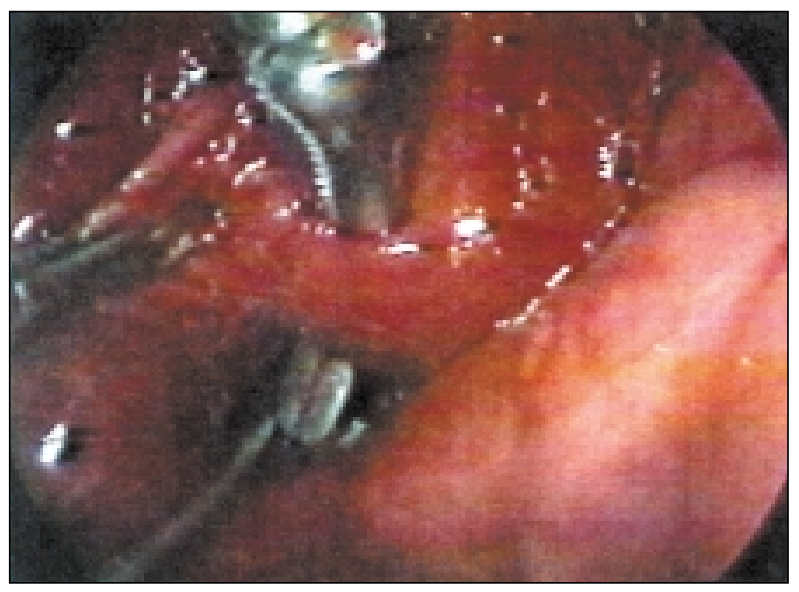

Fig. 2 - Pinça contornando o canal arterial.

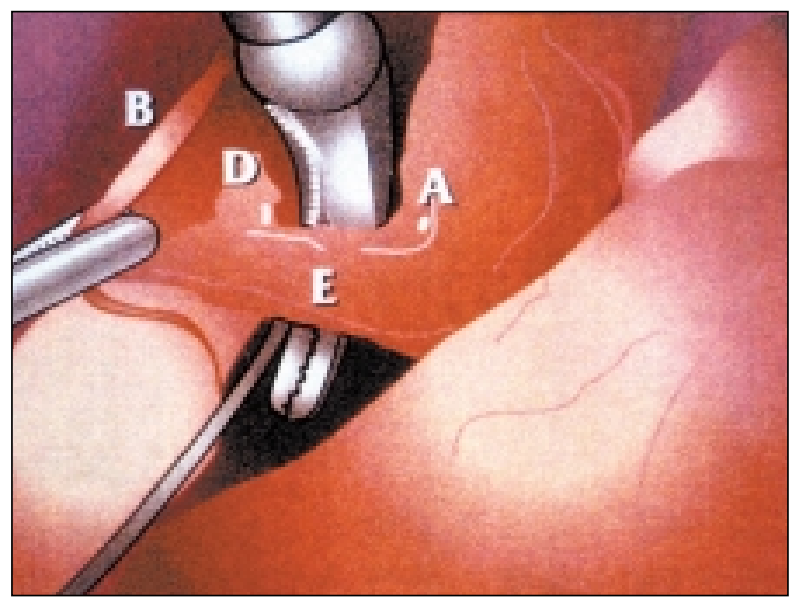

Fig. 3 - Reprodução esquemática da operação. A - aorta, B - nervo vago, D - artéria pulmonar, E - canal arterial. 
Souto G L L, Tinoco R C, Tinoco A C A, Caetano C S, Souza J B, Paula A G, Teixeira M A, Carvalho M R, Botelho A C, Coelho S B P, Souto H B - Ligadura videotoracoscópica da persistência do canal arterial. Rev Bras Cir Cardiovasc 2000; 15(2): $154-9$.

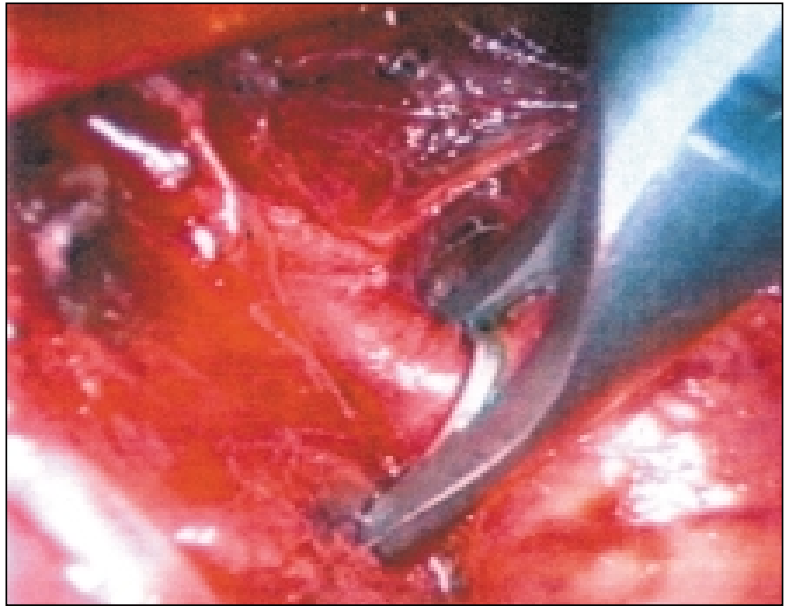

Fig. 4 - Clipagem do canal

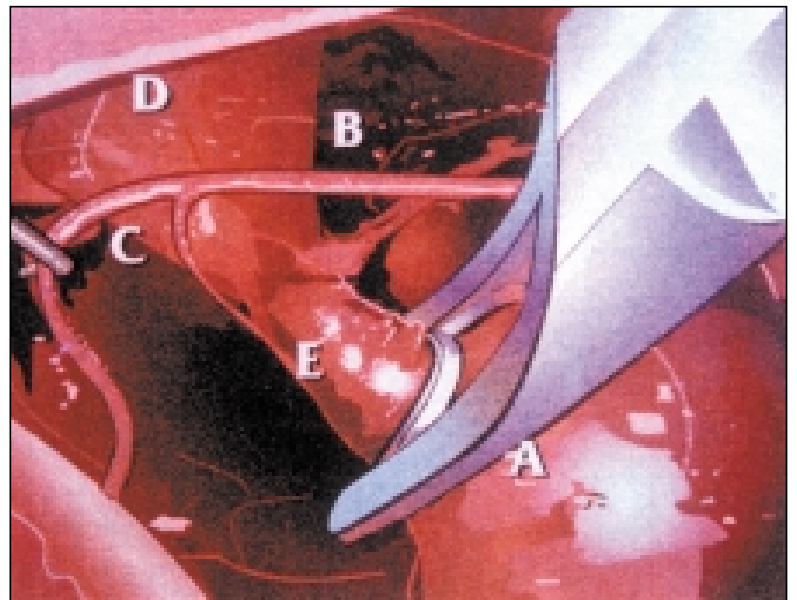

Fig. 5 - Reprodução esquemática da clipagem do canal. A - aorta, B - nervo vago, C - nervo laríngeo, D - artéria pulmonar, E - canal arterial.

canal é largo, com mais de $1 \mathrm{~cm}$ de diâmetro, não podendo ser ligado com clip, que é colocado ou não após a ligadura com fio.

A pleura é deixada aberta e o pulmão expandido sob controle no vídeo, dispensando, assim, o uso de dreno no tórax.

Durante a operação, um cardiologista monitoriza, com estetoscópio esofágico, o desaparecimento do sopro após clipagem ou ligadura do canal com fio.

\section{RESULTADOS}

Obtivemos sucesso em 37 pacientes (92,5\%), sendo necessária a reversão para o procedimento convencional em 3 pacientes (7,5\%).

Não houve óbito e a média de permanência

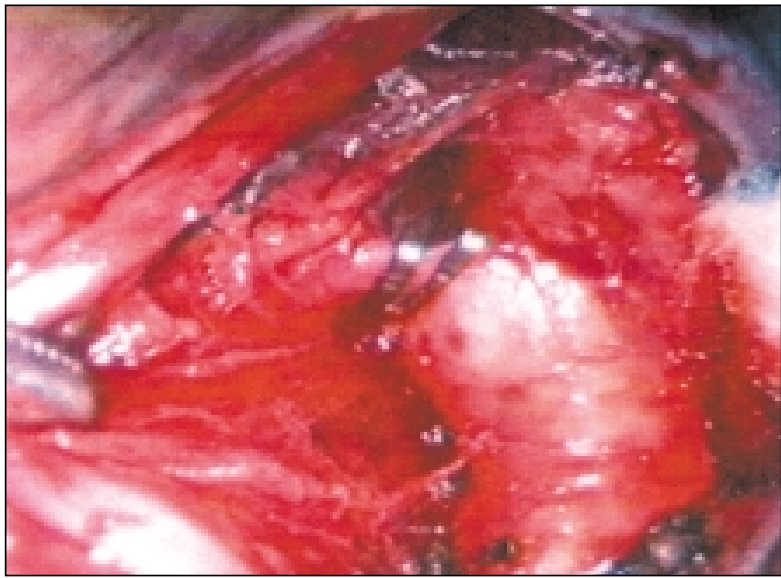

Fig. 6 - Canal arterial clipado duplamente.

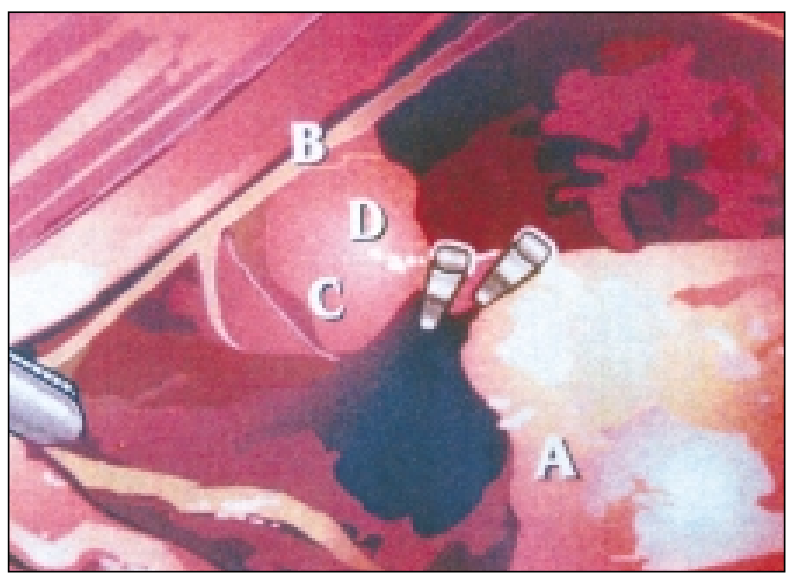

Fig. 7 - Reproducão esquemática do canal já clipado duplamente. A - aorta, B - nervo vago, C - nervo laringeo recurrente, D artéria pulmonar.

hospitalar foi de 48 horas. Até agora, no seguimento desses pacientes, não houve shunt residual e nem reabertura com aparecimento de sopro.

\section{COMENTÁRIOS}

A PCA detectada clinicamente por sopro contínuo e pela ecocardiografia, têm indicação formal de tratamento. Suas contra-indicações são a doença vascular pulmonar oclusiva já instalada e a presença de cardiopatia congênita cianótica canal dependente $(13,16)$

Diversas técnicas e táticas para tratamento têm sido propostas desde a $1^{\circ}$ descrição cirúrgica bem sucedida de GROSS \& HUBBARD ${ }^{(5)}$ em 1939, ligando o canal com fio. Entre estas técnicas estão a secção do canal e sutura dos cotos ${ }^{(17)}$, toracotomia axilar com ligadura extra pleural ${ }^{(18)}$ e, mais recentemente, com a ligadura do canal por videotoracosco- 
Souto G L L, Tinoco R C, Tinoco A C A, Caetano C S, Souza J B, Paula A G, Teixeira M A, Carvalho M R, Botelho A C, Coelho S B

P, Souto H B - Ligadura videotoracoscópica da persistência do canal arterial. Rev Bras Cir Cardiovasc 2000; 15(2): 154-9.

pia $(14,15,19)$, fechamento percutâneo proposto por Rashkind com umbrella $(8,11)$ e fechamento com coil de Gianturco ${ }^{(10)}$. Todas as técnicas propostas buscam menor traumatismo, menor risco para o paciente, menor custo e a mesma efetividade do tratamento cirúrgico convencional, operação com secção e sutura dos cotos com toracotomia clássica.

O tratamento cirúrgico, seja ele feito por toracotomia póstero-lateral esquerda ou transaxilar, tem custo maior, direto e indireto. Direto por maior permanência do paciente no hospital e indireto pelo custo social do procedimento, além da presença do incômodo da dor no pós-operatório e a cicatriz.

O fechamento da PCA percutâneo por cateter, oferece algumas vantagens, já que não há incisão, ou anestesia geral (20), embora a sedação intravenosa seja usual. A permanência no hospital é pequena, em geral \pm 24 horas. O custo é baixo, embora GRAY et al. (21) em 1993, e HAWKINS et al. (18), em 1996, tenham demonstrado a superioridade cirúrgica do fechamento percutâneo com cateter em relação aos resultados e ao custo.

O fechamento da PCA percutâneo por cateter, usando umbrella ou coil, é um tratamento em evolução que ainda não chegou ao ideal. Dentro de suas limitações, estão a persistência do shunt na ordem de $27 \%$ em 6 semanas de seguimento, diminuindo entre $10 \%$ e $20 \%$ em 6 meses. A persistência de shunt aumenta com o tamanho do canal $(13,22)$. A embolização do coil é freqüente $(13,16,23,24)$ e tem sido reportado hemólise ${ }^{(25)}$. A necessidade de profilaxia contra endarterite nos shunt residuais (13) ainda só é aplicável em pacientes com peso corporal maior de $6 \mathrm{~kg}$, devido à ausência de cateteres $(8,11)$. A ocorrência de estenose pulmonar é outro inconveniente. A necessidade de re-intervenção nas umbrella e mesmo nos coils aumenta o custo.

A técnica de ligadura por videotoracoscopia nos pareceu vantajosa atualmente sobre as outras. $O$ tempo de permanência hospitalar dos pacientes operados com videotoracoscopia é baixo, sendo em média de 48 horas. Acreditamos que a experiência com a técnica nos trará maior confiança na realização do procedimento e diminuirá o período de internação. Os últimos pacientes receberam alta hospitalar em 24 horas, reduzindo, assim, ainda mais os custos. Não houve nenhum shunt residual ou lesão do nervo recurrente, embora a literatura mostre $2,1 \%$ de shunt residual e $2,6 \%$ de lesão parcial do nervo recurrente, com recuperação posterior (15).
Nosso tempo cirúrgico diminuiu de 80 minutos para entre 20 a 40 minutos. Não houve complicação no pós-operatório como pneumotórax, sangramento ou quilotórax.

A reversão para operação convencional foi necessária em 3 pacientes. Em 2 casos, houve sangramento ao contornar o canal com a pinça, os quais não estavam bem dissecados. No terceiro caso, houve lesão de uma veia pulmonar junto ao hilo do pulmão. Todas essas complicações, facilmente contornadas, ocorreram nos dez primeiros pacientes. Acreditamos que o canal, ao contrário do que foi preconizado por LABORDE et al. $(14,15)$, deve ser bem dissecado, identificando-se bem as estruturas para posterior clipagem ou ligadura. A dissecção incompleta pode levar a shunt residual e traumatismo do nervo recurrente. Não acreditamos que os canais com mais de $1 \mathrm{~cm}$ sejam limitação à técnica $(14,15)$. Quando encontramos canais acima de $1 \mathrm{~cm}$ de largura, usamos ligadura com fio grosso de algodão antes de usar o clip. A limitação da técnica está nos canais adultos, muitas vezes calcificados ou com paredes extremamente finas, e naqueles indivíduos com sínfise pleural esquerda.

Todos os pacientes foram submetidos a avaliação clínica ecocardiográfica antes da alta hospitalar, sem evidência de permanência de shunt. Estamos acompanhando esses pacientes e, até agora, todos se encontram bem. A aplicabilidade desta técnica é fácil. Quase todos os hospitais já têm aparelho de vídeo para laparoscopia, portanto não há necessidade de aquisição de material especializado é possível, com segurança, obter uma curva de aprendizado rápida.

Não tivemos nenhum caso de PCA em prematuros, pretendemos, no futuro, estender nossas indicações para esta população. Na literatura existem trabalhos mostrando a eficiência da técnica para esses pacientes (19). Não há necessidade de estímulo elétrico na identificação do nervo recurrente; apenas uma boa dissecção resolve.

\section{CONCLUSÃO}

Acreditamos que a ligadura toracoscópica da PCA é um procedimento seguro, pouco traumático, eficaz, com baixo índice de complicações, curva de aprendizado pequena e custo minimizado. 
Souto G L L, Tinoco R C, Tinoco A C A, Caetano C S, Souza J B, Paula A G, Teixeira M A, Carvalho M R, Botelho A C, Coelho S B P, Souto H B - Ligadura videotoracoscópica da persistência do canal arterial. Rev Bras Cir Cardiovasc 2000; 15(2): 154-9.

RBCCV 44205-497

Souto G L L, Tinoco R C, Tinoco A C A, Caetano C S, Souza J B, Paula A G, Teixeira M A, Carvalho M R, Botelho A C, Coelho S B P, Souto H B - Thoracoscopic closure of patent ductus arteriosus (PDA). Rev Bras Cir Cardiovasc 2000; 15(2): 154-9.

ABSTRACT: Background: As the advance of minimally invasive surgical techniques becomes more apparent, new applications are being identified. The present report describes the videothoracoscopy technique for closure of patent ductus arteriosus (PDA).

Objective: To report our initial surgical experience with this procedure.

Methods: From March 1994 to November 1999, thoracoscopic closure of PDA was performed in 40 patients, males and females, whose age ranged from 8 months to 17 years, and whose mean weight was $15 \mathrm{~kg}$. After induction of general anesthesia and intubation, the patient was positioned on right side, as for a posterolateral thoracostomy. Four thoracostomies of 3 to $5 \mathrm{~mm}$ long were made in the left hemithorax, each corresponding in size to the appropriate instruments for work, than the PDA was dissected up and double clipped with titanium clips or closed by a thick suture with intracorporeal knot.

Result: Three patients required conversion to thoracotomy owing to bleeding (7.5\%). There was no operative mortality and the mean hospital stay was 48 hours.

Conclusion: This is a safe procedure, with low complication rate and low cost.

DESCRIPTORS: Cardiac surgical procedures, methods. Arterial channel, surgery. Thoracoscopy, methods. Surgery minimally invasive, methods.

\section{REFERÊNCIAS BIIBLIOGRÁFICAS}

1 Brookmm \& Heymam M A - Patent ductus arteriosus. In: Emmanovildes G C, Riemenchneider T A, Allen H D, Gutgesell H P, eds. Heart disease in infants, children and adolescents, including the fetus and young adult. 5 ed. Baltimore: William \& Wilkins, 1995: $746-64$.

2 Campbell M - Natural history of persistent ductus arteriosus. Br Heart $J$ 1968; 30: 4-13.

Muro J C - Ligation of the ductus arteriosus. Ann Surg 1907; 46: 335-8.

4

Graybiel A, Strieder J W, Bayer N H - An attempt to obliterate the patent ductus arteriosus in a patient with bacterial endocarditis. Am Heart $J$ 1938; 15 621-4.

5 Gross R E \& Hubbard J P - Surgical ligation of a patent ductus arteriosus: a report of first successful case. JAMA 1939; 112: 729-31.

6 Porstmann W, Wierny L, Warnke $\mathrm{H}$ - The closure of the patent ductus arteriosus without thoracotomy. Thoraxchir Vask Chir 1967; 15: 199-203.

7 Porstmann W, Wierny L, Warnke H, Gerstberger G, Romaniuk P A - Catheter closure of patent ductus arteriosus: 62 cases treated without thoracotomy. Radiol Clin North Am 1971; 9: 203-18.

8 Rashkind W J, Mullins C E, Hellenbrand W E, Tait M A - Nonsurgical closure of patent ductus arteriosus: clinical application of the Rashkind PDA occluder system. Circulation 1987; 75: 583-92.
9 Hijazi Z M \& Geggel R L - Results of anterograde transcatheter closure of patent ductus arteriosus using single or multiple Gianturco coils. $A m J$ Cardiol 1994; 74: 925-9.

10 Lloyd T R, Fedderly R, Mendelsohn A M, Sandhu S $\mathrm{K}$, Beekman R H - Transcatheter occlusion of patent ductus arteriosus with Gianturco coils. Circulation 1993; 88 (4 Pt 1): 1412-20.

11 Fontes V F, Pedra C A, Esteves C A et al. - Cierre percutaneo del conducto arterioso con la técnica de Rashkind: experiência inicial del Instituto Dante Pazzanese de Cardiologia, São Paulo, Brasil. Arch Inst Cardiol Mex 1996; 66: 129-37.

12 Nykanen D G, Hayes A M, Benson L N, Freedom R $M$ - Transcatheter patent ductus arteriosus occlusion: application in the small child. J Am Coll Cardiol 1994; 23: 1666-70.

13 Pedra C A C, Esteves C A, Braga S L N, Kambara A, Fontes V F - Oclusão percutânea do canal arterial: estado da arte. Rev Bras Cardiol Inv 1997; 2: 22-5.

14 Laborde F, Noirhomme P, Karam J, Batisse A, Bourel $P$, Saint Maurice O - A new video-assisted thoracoscopic surgical technique for interruption of patent ductus arteriosus in infants and children. $J$ Thorac Cardiovasc Surg 1993; 105: 278-80.

15 Laborde F, Folliguet T, Batisse A, Dibie A, da Cruz E, Carbognani D - Video-assisted thoracoscopic surgical interruption: the technique of choice for patent ductus arteriosus: routine experience in 230 pediatric cases. J Thorac Cardiovasc Surg 1995; 110: 1681-5. 
Souto G L L, Tinoco R C, Tinoco A C A, Caetano C S, Souza J B, Paula A G, Teixeira M A, Carvalho M R, Botelho A C, Coelho S B P, Souto H B - Ligadura videotoracoscópica da persistência do canal arterial. Rev Bras Cir Cardiovasc 2000; 15(2): 154-9.

16 Rao P S \& Sideris E B - Transcatheter occlusion of patent ductus arteriosus: state of the art. $J$ Invasive Cardiol 1996; 8: 278-88.

17 Mavroudis C, Backer C L, Gevitz M - Forty-six years of patent ductus arteriosus division at Children's Memorial Hospital of Chicago: standards for comparison. Ann Surg 1994; 220: 402-10.

Hawkins J A , Minich L L, Tani L Y, Sturtevant J E, Orsmond G S, McGough E C - Cost and efficacy of surgical ligation versus transcatheter coil occlusion of patent ductus arteriosus. J Thorac Cardiovasc Surg 1996; 112: 1634-9.

19 Forster R - Thoracoscopic clipping of patent ductus arteriosus in premature infants. Ann Thorac Surg 1993; 56: 1418-20.

20 Pedra C A, Pedra S R, Esteves C A et al. - Experiência global no fechamento percutâneo do canal arterial. Arq Bras Cardiol 1998; 71: 769-80.

21 Gray D T, Fyler D C, Walker A M, Weinstein M C,
Chalmers T C - Clinical outcomes and costs of transcatheter as compared with surgical closure of patent ductus arteriosus: The Patent Ductus Arteriosus Closure Comparative Study Group. N Engl J Med 1993; 329: 1517-23.

22 Ali Khan M A, al Yousef S, Mullins C E, Sawyer W Experience with 205 procedures of transcatheter closure of ductus arteriosus in 182 patients, with special reference to residual shunts and long-term follow-up. J Thorac Cardiovasc Surg 1992; 104: 1721-7.

23 Moore J W, George L, Kirkpatrick S E - Closure of residual patent ductus arteriosus with occluding spring coil after implant of a Rashkind occluder. Am Heart J 1994; 127(4 Pt 1): 943-5.

24 Lloyd T R, Beekman RH II, Moore $\mathrm{J}$ - Weal the PDA coil registry: report of the first 535 procedures. Circulation 1995; 92: I-380.

25 Pedra C A, Pedra S F, Fontes V F - Hemolysis associated with coil occlusion of the arterial duct. Cardiol Young 1998; 8: 63-6. 